\title{
Una nueva especie de Miconia (Melastomataceae) con anteras connadas de Colombia
}

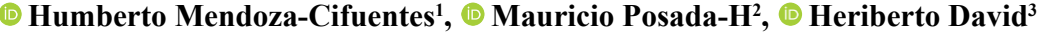 \\ ${ }^{1}$ Instituto Alexander von Humboldt, Herbario FMB, Villa de Leiva, Boyacá, Colombia \\ ${ }^{2}$ Herbario de la Universidad del Valle, CUVC, Cali, Colombia \\ ${ }^{3}$ Herbario de la Universidad de Antioquia, HUA, Medellín, Colombia
}

\begin{abstract}
Resumen
La tribu Miconieae comprende cerca de 1.900 especies neotropicales y en recientes trabajos se propone solo un género para la tribu, Miconia, que incluye la totalidad de los géneros considerados previamente, entre los que se incluye el Tococa. En el presente trabajo se describe e ilustra una nueva especie bajo el género Miconia, que presenta anteras lateralmente connadas, procedente del departamento del Chocó, Colombia, la cual se asocia con las especies de la tribu Miconieae con anteras coherentes tales como Tococa symphyandra y Miconia croatii. Este nuevo taxón se identifica por el indumento de las ramas de tricomas elongados y alveolados, haz foliar bullada, el cáliz cerrado en botones y con grandes dientes dorsales, los pétalos y el estilo más grandes que las anteriores especies, y el ápice del ovario truncado. Crece en áreas muy húmedas de bosque subandino en la región central de la cordillera Occidental y hasta ahora solo se conoce de la localidad tipo. (C) 2019. Acad. Colomb. Cienc. Ex. Fis. Nat.
\end{abstract}

Palabras clave: Bosque subandino; Chocó; Norte de los Andes; Taxonomía; Tococa.

A new species of Miconia (Melastomataceae) with connate anthers from Colombia

\begin{abstract}
The Miconieae tribe comprises about 1,900 Neotropical species and recent studies propose only one genus for the tribe, Miconia, which includes all of the genera previously considered including Tococa. Here we describe and illustrate a new species of the genus Miconia with lateral connate anthers from the department of Chocó, Colombia. This new taxon is associated with the species of the Miconieae tribe with laterally coherent anthers in a ring stamens such as Tococa symphyandra and Miconia croatii. This new taxon is characterized by the indument on branches with elongate and alveolate trichomes, bullate leaves, closed calyx in buds and with large dorsal teeth, bigger petals and style, and a truncated ovary apex. This new species grows in very humid areas of the sub-Andean forest in the central region of the Western Cordillera and at present, it is known only from the type locality. (C) 2019. Acad. Colomb. Cienc. Ex. Fis. Nat.
\end{abstract}

Key words: Chocó; north of the Andes; Sub-Andean forest; Taxonomy; Tococa.

\section{Introducción}

Miconia Ruiz \& Pav. (1794:60), con cerca de 1.900 especies, es el género de plantas leñosas de las angiospermas más diverso con distribución restringida al Neotrópico (Goldenberg, et al., 2008, 2013; Michelangeli, et al., 2016, 2018). Colombia es el país con el mayor número de especies del género, ya que alberga el $36 \%$ de sus taxones, con alrededor de 380 especies (Almeda, et al., 2016).

En su definición tradicional, Miconia se circunscribe como el grupo de especies de la tribu Miconieae que desarrollan inflorescencias terminales y flores con pétalos apicalmente redondeados (Goldenberg, et al., 2013). Este conjunto de caracteres también se presenta en el género Tococa Aublet, que se diferencia de Miconia principalmente por la presencia de mirmecodomacios en ambas hojas y los pétalos emarginados apicalmente (Michelangeli, 2005). Sin embargo, y con base en filogenias moleculares, se reconoce que Miconia es un grupo parafilético, al igual que la mayoría de los géneros más diversos de la tribu (Michelangeli, et al., 2004, 2008; Goldenberg, et al., 2008), razón por la cual se ha propuesto incluir a Tococa, así como a la mayoría de los géneros de Miconiaeae, dentro de Miconia (ver Michelangeli y Meier, 2013; Gamba y Almeda, 2014; Michelangeli, et al., 2016, 2018).

\footnotetext{
*Correspondencia:

Humberto Mendoza Cifuentes; hmendoza@humboldt.org.co

Recibido: 4 de marzo de 2019

Aceptado: 7 de junio de 2019

Editor: Elizabeth Castañeda
} 
La presente novedad taxonómica se asocia con dos especies del género Tococa, T. symphyandra (Triana) Cogn. y T. croatii Almeda, esta última recientemente transferida a Miconia croatii (Almeda) Michelang. \& Almeda (Michelangeli, et al., 2018), ya que comparten el carácter de anteras connadas. Dentro de la tribu Miconieae, las anteras connadas que forman un anillo solo se conocen para estos tres taxones (Almeda, 1989; Michelangeli, 2005). En consecuencia, esta nueva especie se describe bajo el género Miconia, pero relacionándola con una especie ya transferida a este género y con una del género Tococa que aún no ha sido restituida oficialmente al género Miconia.

\section{Materiales y métodos}

Se recolectaron en campo individuos de la familia Melastomataceae en zonas inexploradas del departamento de Chocó, ubicadas en el centro de la cordillera Occidental de Colombia, en busca de novedades taxonómicas. Se revisó la literatura pertinente a los géneros Miconia y Tococa, la base de datos del herbario COL (http://www.biovirtual.unal. edu.co/es/colecciones/search/plants/), el catálogo de Melastomataceae de Colombia (Almeda, et al., 2016) y la flora mesoamericana (Almeda, 2009). Una vez identificada la novedad taxonómica, se procedió a confrontar directamente las colecciones del presente hallazgo con colecciones de los herbarios Nacional Colombiano (COL), de la Universidad de Antioquia (HUA), la Universidad del Valle (CUVC) y el Federico Meden Bogotá (FMB). Además, se consultaron las imágenes tipo de la totalidad de las especies de Miconia y Tococa de Colombia en la base de datos de Global Plants JSTOR (http://plants.jstor.org/search?plant).

Una vez definido este taxón como una nueva especie, se la describió e ilustró y se establecieron sus límites morfológicos con respecto a las especies más afines dentro de los géneros Miconia y Tococa. Para la descripción se midieron las partes vegetativas y de la inflorescencia en material seco de herbario utilizando un calibrador digital de $0,01 \mathrm{~mm}$ de precisión (Mitutoyo Serie 500). Las medidas de las partes florales se basaron en flores frescas preservadas en etanol al $70 \%$ y se establecieron utilizando un microscopio Leica S8AP0. Los tricomas se fotografiaron con una cámara MC190 HD a partir de material seco para las estructuras vegetativas y de material fresco conservado en etanol para las partes florales; posteriormente, se clasificaron con base en el atlas de tricomas de Melastomataceae de Wurdack (1986).

\section{Resultados}

Miconia cornifera Humberto Mend., Posada-Herrera \& Heriberto David, sp. nov. (Figuras 1, 2).

TIPO: COLOMBIA. Chocó: San José del Palmar, escuela antigua de Galápagos, trocha que conduce de la escuela a las cascadas, $1.300-1.600 \mathrm{~m}, 4^{\circ} 50^{\prime} 1$ ' $\mathrm{N}$, 76¹1'33'O, 26-31 may 2008 (fl), H. Mendoza 17327 (Holótipo: FMB; Isótipos: COL, FMB, HUA, CUVC).
Diagnosis. The new species resembles Tococa symphyandra and Miconia croatii but differ from them by the indumentum on branchlets and petioles densely lanose with brownish elongated alveolate trichomes, upper leaf blade bullate-setulose, closed calyx in buds with long dorsal teeth, petals 15.9-18.8 mm long, style 13.4-14.3 mm long, and truncate ovary apex.

Arbusto de 1,5 a 2,0 m de altura. Ramas distales subcuadrangulares, entrenudos de 2 a $3 \mathrm{~cm}$ de largo. Indumento sobre ramas y peciolo densamente lanoso compuesto de tricomas elongado-alveolados curvos o retorcidos en muestras secas (tipo12 de Wurdack, 1986) de color marrón y de 0,5 a $1 \mathrm{~mm}$ de largo; ejes de la inflorescencia con indumento denso de tricomas elongado-alveolados (tipo 12) y, además, tricomas glandulares estipitados (tipo 1) de 0,7 a $1,3 \mathrm{~mm}$ de largo; hipanto-cáliz con indumento denso de tricomas glandulares estipitados (tipo 1) y tricomas elongadoalveolados curvos (tipo 12) de 0,8 a $1,2 \mathrm{~mm}$ de largo. Hojas de un mismo nudo de tamaño similar o levemente desiguales, sin mirmecodomacios. Pecíolos de 2,5 a 10,0

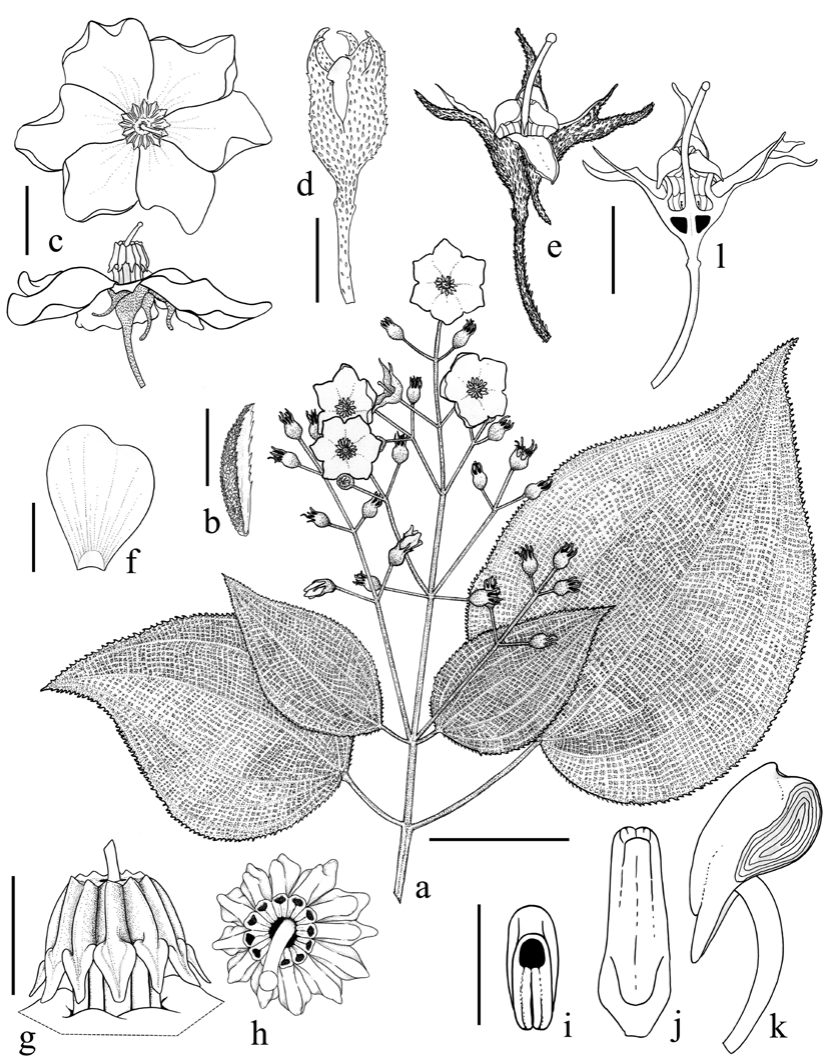

Figura 1. Miconia cornifera. a. Rama florífera. b. Bráctea. c. Flor en vista superior y lateral. d. Botón floral. e. Hipanto-cáliz en vista lateral. f. Pétalo. g. Estambres connados en vista lateral. h. Estambres connados en vista superior. i. Ápice de la antera. j. Antera en vista dorsal. k. Estambre individual extraído y en vista lateral. l. Corte longitudinal del hipanto-ovario y del estilo. Escalas: $5 \mathrm{~cm}$ en $\mathbf{a} ; 5 \mathrm{~mm}$ en $\mathbf{b}, \mathbf{g}, \mathbf{h} ; 10 \mathrm{~mm}$ en $\mathbf{c}, \mathbf{d}, \mathbf{e}, \mathbf{f} ; 2 \mathrm{~mm}$ en $\mathbf{i}, \mathbf{j}, \mathbf{k}$. Ilustración: Angélica Ramírez Mendoza 
$\mathrm{cm}$ de largo, teretes hacia la base y ligeramente caniculados hacia el ápice. Lámina foliar de 11,5 a 21,0 × 9,0-15,5 $\mathrm{cm}$, anchamente ovada, delgada y membranácea; margen dentada; base cordada; ápice acuminado; haz densamente bullado-setulosa, tricomas (tipo 15) de 0,4 a 0,6 mm de largo; envés con indumento lanoso denso a laxo sobre los nervios, tricomas elongado-alveolados (tipo 12) de 0,4 a 0,6 mm de largo. Nervadura con 3 a 4 pares de venas secundarias basales acompañando la vena media. Inflorescencia terminal, paniculada, de 20 a $24 \mathrm{~cm}$ de largo, sésil o con un pedúnculo de 2,5 a 4,5 cm de largo, con 25 a 40 flores pediceladas; eje central cuadrangular, con 5 a 6 nudos de ramificación; paracladios basales de 10 a $12 \mathrm{~cm}$ de largo con un nivel de ramificación; ápices de las ramas trifloros; brácteas semejantes a hojas pequeñas en los nudos basales, ovadas, de 6,7 a $10,0 \times 3,9$ a 4,5 cm, con un pequeño pecíolo de 0,4 a 2,0 cm de largo; bracteolas elípticas a estrechamente elípticas, de 5,8 a $9,9 \times 2,0$ a $2,2 \mathrm{~mm}$, tempranamente

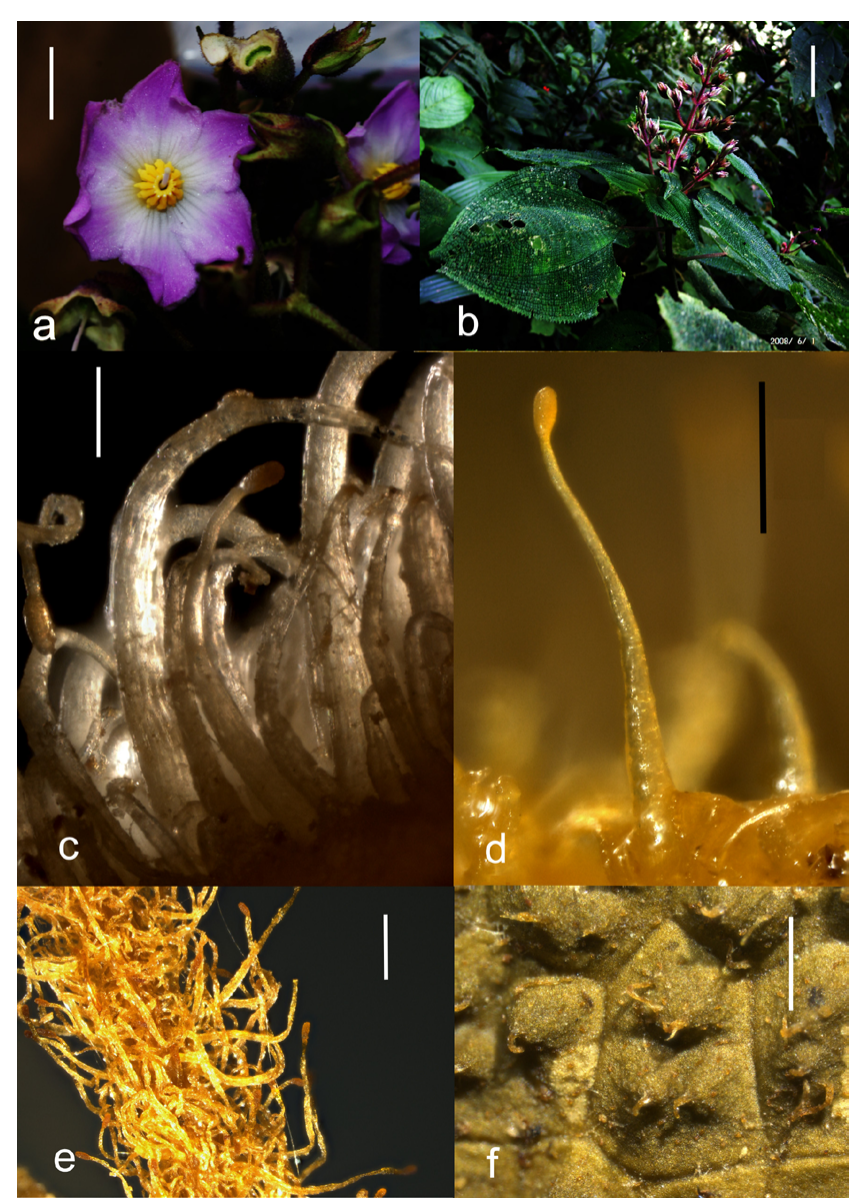

Figura 2. Imágenes de campo y tricomas de Miconia cornifera. a. Vista de flores. b. Rama florífera. c. Tricomas del eje de la inflorescencia (a partir de muestras frescas almacenadas en etanol). d. Tricoma glandular del eje de la inflorescencia (a partir de muestras frescas almacenadas en etanol). e. Tricomas del eje de la inflorescencia (a partir de material seco). f. Tricomas del haz foliar. Escalas: $10 \mathrm{~mm}$ en $\mathbf{a} ; 5 \mathrm{~cm}$ en $\mathbf{b} ; 0,2 \mathrm{~mm}$ en $\mathbf{c} ; 0,5 \mathrm{~mm}$ en $\mathbf{d}, \mathbf{e}, \mathbf{f}$. caducas, abaxialmente glabras, adaxialmente con indumento denso de tricomas similares a los de las ramas. Flores de 5 a 6 meras, diplostemonas; pedicelos de 6,5 a 25,2 mm de largo, con indumento denso similar al de la inflorescencia. Hipanto en la antesis en forma de copa, de 3,9 a 6,3 × 6,9 a 7,4 mm; parte libre del ovario de 1,0 a 1,7 mm de largo; internamente glabro. Cáliz cerrado en botones y coronado por un apículo de 3,5 a 4,0 mm de largo, que se abre irregularmente en 2 a 5 lóbulos triangulares de 5,5 a 7,5 mm de largo, estos lóbulos son caducos en frutos inmaduros; dientes dorsales subulados de 4,0 a 7,2 mm de largo, apicalmente curvos, excediendo la longitud de los lóbulos. Corolas patentes; pétalos de 15,9 a $18,8 \times 12,8$ a $15,3 \mathrm{~mm}$, anchamente obovados, apicalmente sigmoideo-emarginados, glabros, color violeta claro y blancuzcos hacia la base en la superficie abaxial, con la margen entera. Estambres isomorfos, con las anteras lateralmente connadas que forman un anillo radialmente simétrico alrededor del estilo de color amarillo; filamentos glabros, aplanados, de 3 a $4 \times 0,8$ a $0,9 \mathrm{~mm}$; anteras oblongas, lateralmente aplanadas, de 2,2 a 3,2 × 1,8 a 2,2 $\mathrm{mm}$ y 1,0 a $1,9 \mathrm{~mm}$ de grosor, ápice truncado-emarginado, con un poro apical de 0,4 a $0,5 \times 0,3$ a $0,4 \mathrm{~mm}$; conectivo engrosado dorsalmente, prolongado en la base dorsal en una estructura triangular o romboide de 1,3 a 2,8 $\times 1,4$ a 1,9 mm. Ovario ínfero, 5-locular, de 2,8 a 3,7 mm de largo; ápice truncado, con algunos tricomas glandular-estipitados rodeando la base del estilo de 0,8 mm de largo; placentas ca. de 1,5 ×0,8 mm, oblongas, con rudimentos seminales en todas las superficies. Estilo recto o levemente curvo, de 13,4 a $14,3 \times 0,6$ a 0,9 $\mathrm{mm}$, diminutamente papilado en su mitad superior, que sobresale conspicuamente del anillo de estambres; estigma levemente expandido, de 0,8 a 1,3 mm de diámetro. Frutos maduros y semillas no vistos.

Etimología. El epíteto específico significa "Miconia con cuernos" y alude a los grandes dientes dorsales del cáliz.

Distribución y hábitat. Conocida solo de la localidad tipo en la vertiente occidental que drena hacia la planicie del Pacífico de la cordillera Occidental. Crece en bosque subandino bajo condiciones de sombra y al lado de caídas de cascadas. La vegetación en el sitio de recolección corresponde a bosques entresacados donde se ha extraído la mayoría de las arboles maderables. El clima de la localidad tipo en el cerro Galápagos se clasifica como frío y muy húmedo, con temperatura promedio de $14^{\circ} \mathrm{C}$, una precipitación anual de más de $4.000 \mathrm{~mm}$ y una humedad relativa que oscila entre 60 y $70 \%$ (Urrutia, 2017).

Fenología. La especie fue recolectada con flores en mayo.

\section{Discusión}

Esta especie se reconoce por el indumento denso en ramas con tricomas elongados y alveolados, haz foliar bulladosetulosa, el cáliz cerrado en botones con grandes dientes dorsales, los pétalos de 15,9 a 18,8 mm de largo, estambres connados por las anteras formando un anillo, el estilo de 13,4 a 14,3 mm de largo, y el ápice del ovario truncado. 
Tabla 1. Comparación de caracteres de Miconia cornifera, M. croatii y Tococa symphyandra. Los tipos de tricomas se basan en Wurdack (1986).

\begin{tabular}{|c|c|c|c|}
\hline \multirow[t]{2}{*}{ Carácter } & \multicolumn{3}{|c|}{ Especie } \\
\hline & Miconia cornifera & Miconia croatii & Tococa symphyandra \\
\hline Indumento y tricomas de las ramas & $\begin{array}{l}\text { Denso, tricomas curvos o } \\
\text { retorcidos de tipo } 12 \mathrm{de} \\
0,5-1,0 \mathrm{~mm} \text { de largo }\end{array}$ & $\begin{array}{c}\text { Laxo a semidenso, tricomas } \\
\text { erectos de tipo } 11 \mathrm{de} \\
1,0-2,0 \mathrm{~mm} \text { de largo }\end{array}$ & $\begin{array}{l}\text { Laxo, tricomas erectos } \\
\text { de tipos } 1 \text { y } 11 \mathrm{de} \\
5,0-15,0 \mathrm{~mm} \text { de largo }\end{array}$ \\
\hline Indumento y tricomas del haz foliar & $\begin{array}{l}\text { Denso, tricomas de tipo } 15 \\
\text { de } 0,4-0,6 \mathrm{~mm} \text { de largo }\end{array}$ & $\begin{array}{l}\text { Laxo, tricomas tipo } 11 \text { de } \\
0,5-2,0 \mathrm{~mm} \text { de largo }\end{array}$ & $\begin{array}{l}\text { Laxo, tricomas de tipo } 11 \\
\text { de } 0,5-2,0 \mathrm{~mm} \text { de largo }\end{array}$ \\
\hline Indumento y tricomas del hipanto & $\begin{array}{c}\text { Denso, tricomas curvos de } \\
\text { tipo } 1 \text { y } 12 \text { de } 0,8-1,2 \mathrm{~mm} \\
\text { de largo }\end{array}$ & $\begin{array}{l}\text { Denso, tricomas erectos } \\
\text { tipo } 1 \mathrm{ca} .0,5 \mathrm{~mm} \text { de largo y } \\
\text { tricomas tipo } 4 \text { (sésiles) }\end{array}$ & $\begin{array}{l}\text { Glabro o pubérulo, } \\
\text { tricomas erectos de tipo } \\
1 \text { ca. } 1,5 \mathrm{~mm} \text { de largo }\end{array}$ \\
\hline Longitud del hipanto (mm) & $3,9-6,2$ & $2,0-2,5$ & $2,5-3,5$ \\
\hline Tipo de cáliz & Cerrado en botones & Cerrado en botones $6,0-9,0$ & Lobulado \\
\hline Longitud dientes dorsales del cáliz (mm) & $4,7-7,2$ & $0,25-0,50$ & $0,25-0,50$ \\
\hline Longitud de los pétalos (mm) & $15,9-18,8$ & $5,0-6,0$ & $6,0-9,0$ \\
\hline Longitud del filamento (mm) & $3,0-4,0$ & $2,5-3,5$ & 3,5 \\
\hline Longitud de las tecas (mm) & $2,2-3,2$ & $1,5-2,1$ & $2,0-2,5$ \\
\hline $\begin{array}{l}\text { Longitud del apéndice conectival basal- } \\
\text { dorsal }(\mathrm{mm})\end{array}$ & $1,3-2,8$ & $1,0-1,5$ & $1,0-1,5$ \\
\hline Ápice del ovario & Truncado & Redondeado & Redondeado \\
\hline Longitud del estilo (mm) & $13,4-14,3$ & $5,0-8,5$ & $6,0-9,5$ \\
\hline
\end{tabular}

Las especies más cercanas, Tococa symphyandra y Miconia croatii, tienen ramas con indumentos laxos de tricomas erectos, las hojas no son ampollado-bulladas por el haz, las flores son mucho más pequeñas, al igual que los dientes dorsales de cáliz (ver comparación y medidas en la Tabla 1). Además, se diferencia de $T$. symphyandra por la ausencia de mirmecodomacios.

Miconia cornifera conforma un grupo con las dos anteriores especies, único por sus anteras unidas formando un anillo, carácter no presente en otras especies de la tribu Miconieae. La disección de las anteras en $M$. cornifera indica que hay tejidos que las conectan lateralmente, es decir son soldadas, por lo que el término más apropiado para esta condición es connado. T. symphyandra y $M$. croatii tienen la misma condición de las anteras de $M$. cornifera.

Vegetativamente, estos tres taxones se parecen a especies con hábito herbáceo y hojas membranáceas tales como Miconia cordifolia Wurdack, M. acalephoides Naudin y $M$. subalpina Gleason, no obstante, estas últimas especies no presentan anteras connadas.

La reciente propuesta de Michelangeli, et al. (2018) en la cual transfieren la mayoría de las especies dentro de la tribu Miconieae al género Miconia, incluidas las especies de Tococa, ha generado controversia debido al gran número de cambios de nomenclatura propuestos, pero, especialmente, por las evidencias en las que se sustentan los autores de la propuesta. Actualmente no existe un estudio integral morfológico y molecular de toda la tribu y las hipótesis filogenéticas publicadas se basan en pocos marcadores moleculares y no consideran la totalidad de las especies tipos de los géneros involucrados, incluido el de Miconia. Es posible que aparezcan nuevas hipótesis basadas en muestras más grandes y mayor número de marcadores moleculares que seguramente variarán notoriamente de las ya publicadas y establecerán nuevas configuraciones internas en la tribu, permitiendo otras posibilidades para establecer grupos naturales.

El grupo que involucra a $M$. cornifera se asocia más a la circunscripción clásica de Miconia que a la de Tococa, aspecto que fue documentado por Michelangeli (2005) al establecer que T. croatii y T. symphyndra no conforman el grupo de Tococa sensu stricto. Por esta razón, este grupo de tres especies queda mejor ubicado dentro de Miconia, aunque debe subrayarse que los autores no se adhieren completamente a las nuevas propuestas de nomenclatura en la tribu Miconieae.

\section{Agradecimientos}

Expresamos nuestros sinceros agradecimientos al Instituto Alexander von Humboldt por el apoyo en el uso de equipos y laboratorios en la sede de Villa de Leyva, a la administración de los herbarios COL, HUA y FMB y a los revisores anónimos por sus aportes al texto. 


\section{Contribución de los autores}

Humberto Mendoza-Cifuentes: autor principal y receptor de correspondencia, compilación de la información, recolecciones, y escritura general del artículo; Mauricio Posada: escritura general del artículo; Heriberto David: escritura general del artículo, revisión de colecciones

\section{Conflicto de intereses}

Los autores declaran no tener conflictos de intereses.

\section{Referencias}

Almeda, F. (1989). Five new berry-fruited species of tropical American Melastomataceae. Proc. Calif. Acad. Sci. 46 (5): 137-150.

Almeda, F. (2009). Melastomataceae. En G. Davidse, M. SousaSánchez, S. Knapp, F. Chiang (Editores), Cucurbitaceae a Polemoniaceae. Fl. Mesoamer. 4 (1): 164-338.

Almeda, F., Alvear, M., Mendoza-C., H, Penneys. D.S., Michelangeli, F. (2016). Melastomataceae. En R. Bernal, S.R. Gradstein, M. Celis (Editores), Catálogo de plantas y líquenes de Colombia. p. 1585-1664. Bogotá, Colombia: Editorial Universidad Nacional de Colombia. http:// catalogoplantasdecolombia.unal.edu.co/es/resultados/ familia/Melastomataceae/

Gamba, D., Almeda, F. (2014). Systematics of the Octopleura Clade of Miconia (Melastomataceae: Miconieae) in Tropical America. Phytotaxa. 179 (1): 1-174. Doi: 10.11646/ phytotaxa.179.1.1

Goldenberg, R., Penneys, D.S., Almeda, F., Judd, W.S., Michelangeli, F.A. (2008). Phylogeny of Miconia (Melastomataceae): Patterns of stamen diversification in a megadiverse Neotropical genus. Int. J. Plant Sci. 169 (7): 963-979. https://www.journals.uchicago.edu/ doi/10.1086/589697

Goldenberg, R., Almeda, F., Caddah, M.K., Martins, A.B., Meirelles, J., Michelangeli. F.A., Weiss, M. (2013). Nomenclator botanicus for the neotropical genus Miconia (Melastomataceae: Miconieae). Phytotaxa. 106: 1-171. Doi: $10.11646 /$ phytotaxa.106.1.1
Michelangeli, F.A. (2005). Tococa (Melastomataceae). Flora Neotrop. 98: 1-114.

Michelangeli, F.A. \& Meier, W. (2013). A new anisophyllous species of Miconia (Melastomataceae: Miconieae) from the Coastal Cordillera in northern Venezuela. Phytotaxa. 79 (1): 37-44. Doi: 10.11646/phytotaxa.163.3.3

Michelangeli, F.A., Penneys, D.S., Giza, J., Soltis, D., Hils, M.H., Skean, J.D. (2004). A preliminary phylogeny of the tribe Miconieae (Melastomataceae) based on nrITS sequence data and its implications on inflorescence position. Taxon. 53: 279-290. Doi: 10.2307/4135608

Michelangeli, F.A., Judd, W.S., Penneys, D.S., Skean, J.D., Bécquer-Granados, E.R., Goldenberg, R., Martin, C.V. (2008). Multiple events of dispersal and radiation of the tribe Miconieae (Melastomataceae) in the Caribbean. Botanical Review. 74: 53-77. https://link.springer.com/ article/10.1007\%2Fs12229-008-9004-x

Michelangeli, F.A., Almeda, F., Alvear, M., Bécquer, E.R., Burke, J., Caddah, M.K., Goldenberg, R., Ionta, G.M., Judd, W.S., Majure, L.C., Meirelles, J., Nicolas, A.N., Ocampo, G., Penneys, D.S., Dan Skean Jr., J., UlloaUlloa, C. (2016). (2462) Proposal to conserve Miconia, nom. cons. against the additional names Maieta and Tococa (Melastomataceae: Miconieae). Taxon. 65 (4): 892-893.

Michelangeli, F.A., Goldenberg, R., Almeda, F., Judd, W.S., Bécquer, E.R., Ocampo, G., Ionta, G.M., Dan Skean Jr., J., Majure, L.C., Penneys, D.S. (2018). Nomenclatural novelties in Miconia (Melastomataceae: Miconieae). Brittonia. First online: 1-40. Doi: 10.1007/s12228-0189546-0

Urrutia, N.S. (2017). Herpetofauna del Alto Galápagos, San José del Palmar, Chocó. Instituto de Investigaciones Ambientales del Pacífico John Von Neumann (IIAP). Dataset. Fecha de consulta: 9 de diciembre, 2017. Disponible en: http://doi. org/10.15472/ijpswu

Wurdack, J.J. (1986). Atlas of hairs for Neotropical Melastomataceae. Smithson. Contrib. Bot. 63: 1-80. 